\title{
Rivista Sperimentale di Freniatria
}

La rivista della salute mentale

THE ITALIAN JOURNAL OF MENTAL HEALTH

VOL. CXLIV - N 1,2020

Susanna Vezzadini

Vittime e società: per la ricostruzione delle reti fiduciarie violate

Susanna Pietralunga, Claudia Salvioli

Aspetti criminologici e giuridici sulla vittima, con particolare riguardo al minore

Heidi Stöckl, Ecaterina Balica, Consuelo Corradi,

Anna Costanza Baldry†, Monika Schröttle,

Belén Sanz-Barbero, Carmen Vives-Cases

Issues in measuring and comparing the incidence of intimate partner

homicide and femicide - A focus on Europe

Anna Maria Scapicchio, Maria Gloria Gleijeses

Trasmissione intergenerazionale dell'abuso e terapia EMDR

Lorenzo Natali

Understanding environmental victimization from a green

criminological perspective

Giovanni Mierolo

I servizi di victim support. L'esperienza di Rete Dafne 


\section{Gabriella Lo Cascio}

"In viaggio nella malattia"

RECENSIONI

NORME REDAZIONALI
La vittimizzazione è una condizione di tipo processuale caratterizzata da un crogiolo di inestricabili dinamiche relazionali vittima-perpetratore, di vulnerabilità, sofferenza, vissuti ed emozioni cui non sono estranee rappresentazioni socialmente determinate. La complessità della condizione vittimale, esposta a proiezioni e meccanismi difensivi collettivi, pregiudizi e manipolazioni, alimenta il rischio di una recursività impotente e dolorosa, che può trasformare profondamente e stabilmente l'identità cristallizzando il ruolo sociale e condizionando la percezione del mondo e delle relazioni come luogo insicuro.

Già il Libro di Giobbe descrive il ritrovarsi vittima senza colpa di eventi negativi e la difficoltà di trovare un senso a quanto accade. Nella logica veterotestamentaria questo pone il problema di come Dio premi o castighi le azioni degli uomini e se sia prevista una riparazione individuale o collettiva, terrena o ultraterrena.

Giobbe testimonia attraverso la propria vita che esistono giusti che soffrono crudelmente. Il lettore sa che i suoi mali vengono da Satana e non da Dio e che sono una messa alla prova della sua fedeltà, ma Giobbe non lo sa, né lo sanno i suoi amici. Quale potrà essere allora la spiegazione? Essi avanzano risposte convenzionali: la felicità degli empi è di breve durata; la disgrazia del giusto saggia la sua virtù; la pena castiga colpe commesse per ignoranza o debolezza.

Contro la rigida correlazione colpa-punizione, Giobbe si solleva con tutta la forza della sua innocenza. Non nega la riparazione terrena, la attende anzi e Dio alla fine gliela concederà, ma per lui è una sofferenza in più che tale riparazione gli venga rifiutata nel presente e si affanna a cercare invano il senso della prova cui è sottoposto. Lotta disperatamente per ritrovare un Dio che si nasconde e che egli si ostina a credere buono. La risposta di Dio non risolverà il problema, rivelerà solo la trascendenza del suo essere e dei suoi disegni e ridurrà alla fine Giobbe al silenzio. 


\section{Aspetti criminologici e giuridici sulla vittima, con particolare riguardo al minore}

\section{Criminological and legal aspects of the victim. Afocus on minor victims}

Susanna Pietralunga*, Claudia Salvioli**

Riassunto: la criminologia ha storicamente incentrato l'attenzione sull'autore del reato, rivolgendo per lungo tempo scarsa attenzione alla vittima. Lo sviluppo della vittimologia ha stimolato un risveglio di riflessioni sulle differenti tipologie e sul ruolo della vittima, mentre - e nonostante l'attenzione rivolta ad essa negli atti internazionali - si continua a registrare scarsità di previsioni normative a sua tutela nel diritto interno. Ne deriva l'importanza di indirizzi di politica criminale e di orientamenti legislativi de jure condendo che riconoscano alla vittima, soprattutto se minore, una tutela paritaria rispetto a quella dell'autore di reato.

Parole Chiave: vittima, vittima minore, tutela normativa della vittima, criminologia, vittimologia.

Abstract: Historically criminology focused its attention on the offender and less on the victim. The development of victimology enhanced the study on different types of victims and on the role of the victim. However, in Italy - despite the attention paid to the victim by International Law - this person is still inadequately protected. Hence, the importance of criminal policy guidelines and legislative policy orientation to grant equal protection to the offender and to the victim, especially if the victim is a minor.

Key Words: victim, minor victim, law to protect the victim, criminology, victimology.

"Professore Associato di Criminologia e Criminologia minorile, Dipartimento di Educazione e Scienze Umane, Università degli Studi di Modena e Reggio Emilia.

** Specialista e Dottore di ricerca in Criminologia clinica, Funzionario della professionalità pedagogica, Centro di Giustizia Minorile di Bologna, Ministero della Giustizia.

Ricevuto 06.02.2020 Accettato 29.02.2020. Submitted 06.02.2020 Accepted 29.02.2020

RSF (ISSN 1129-6437, ISSNe 1972-5582), VOL. CXLIV, 2020, 


\section{L'attenzione verso la vittima nella letteratura criminologica e nelle previsioni normative}

Nei confronti della vittima del reato la letteratura scientifica criminologica è connotata da una scarsa attenzione che si è protratta per lungo tempo: vale la pena di richiamare, circa la genesi di questo nuovo interesse, l'importante impulso derivante dalla riflessione svolta da von Hentig [1] ed individuata come il fondamento della vittimologia, scienza di carattere empirico definita come la "... disciplina che studia il crimine dalla parte della vittima con scopi diagnostici, preventivi, riparativi e trattamentali del reato e della conseguente vittimizzazione" [2].

A tale scarsa attenzione fanno da contraltare previsioni normative altrettanto limitate sotto il profilo del diritto penale, sostanziale e processuale, talché a tutt'oggi occorre rivolgersi agli Atti internazionali per trovare i riferimenti più ampi sulla vittima e sulla sua tutela.

Gli obiettivi e le dimensioni di questa riflessione non consentono una esposizione esaustiva dei riferimenti normativi relativi alla vittima nel diritto interno e negli interventi internazionali, ma una loro sintetica rassegna è essenziale per l'inquadramento del tema in esame.

È opportuno in primo luogo premettere la differente prospettiva adottata sulla vittima dal legislatore rispetto a quella abbracciata dalla riflessione scientifica criminologica e vittimologica: si osserva al riguardo che il diritto positivo valuta le relazioni tra delinquente e vittima in termini statici e nell'ambito di un sistema causa-effetto, non in un modello relazionale ed, in questo senso, la vittima acquisisce rilievo per una sua qualità o per un comportamento oggettivo nei confronti della gente, non per le interazioni con lo stesso, culminate nel fatto reato [3].

In funzione di tale indirizzo, i riferimenti alla vittima previsti dal codice penale costituiscono dei fattori che concorrono ad integrare la realtà empirica individuata dalla previsione normativa; in tal modo la vittima assume rilevanza prevalentemente in rapporto ai suoi comportamenti oggettivi verso l'autore del reato $o$ alle sue qualità, che rilevano in rapporto alla qualificazione del fatto come illecito penale, ma anche per la configurabilità delle circostanze (art. 61 numero 7 e 10, 522 comma 2, 523 comma 2, 576 e 577 c.p.) ${ }^{[4]}$. Rilevano, al riguardo, fattori indicati dalla norma quali l'età della vittima (art. 519, comma 2, n.1 e 2; art 523, art 524, art 530, art 570, art 572, art 591, art 593 c.p.), il sesso (art. 522 comma 1, 523, 526 c.p.), e l'infermità (art 643, art 591 -593, art 579 c.p.).

Talvolta la fattispecie incriminatrice prevede la condotta della vittima come l'antecedente logico formale della condotta del reo, che ne condiziona la tipicità, ed essa dunque assume l'aspetto di presupposto del reato (art 316 c.p.) [3].

Segnatamente, in alcune fattispecie il codice penale attribuisce specifica rilevanza al contributo dato dalla vittima alla realizzazione del fatto di reato: si tratta della provocazione (art 62, numero 2), del concorso del fatto doloso della persona offesa alla determinazione dell'evento ( $\operatorname{art} 62 \mathrm{n}$ 5), del consenso dell'avente diritto (art 50 c.p.), della legittima difesa (art 52 c.p.), e della reazione legittima all'atto arbitrario del pubblico ufficiale (art 4 d.lg. It 14 settembre 1944, n 288) [4].

Del pari, alla base di specifiche fattispecie di reato commesse ai danni di un pubblico ufficiale, di rappresentante di un corpo politico, di un magistrato in udienza ed altri, si colloca lo status socio-professionale della vittima (art 336, 337, 338, 341, 342, 343, 276-278, 295, 296 c.p.), e la presenza di vincoli familiari contraddistingue reati come l'incesto (art. 564 c.p.), la violazione degli obblighi di assistenza familiare (art. 570 c.p.), l'abuso di mezzi di correzione e disciplina (art. 571 c.p.), i maltrattamenti in famiglia (art. 572c.p.)[3].

Anche per ciò che concerne il codice di procedura penale va sottolineato che non si rinviene al suo interno una definizione della vittima, anche se ne vengono indicati gli ambiti di intervento, le facoltà ed i diritti negli artt. $90 \mathrm{e}$ ss. c.p.p.

In esso, il termine vittima è indicato nell'articolo 468 comma 4 ter c.p.p. (modalità protette di escussione del teste): in questo caso la norma fa riferimento al "minore vittima del reato ovvero del maggiorenne infermo di mente vittima del reato". Ancora, un riferimento alla vittima si rinviene nell'articolo 316 comma 1bis c.p.p. inserito dall'art 3, 1. 11 gennaio 2018 numero 4, recante "Modifiche al codice civile, al codice penale, al codice di procedura penale e altre disposizioni in favore degli orfani per crimini domestici", in rapporto alla possibilità di disporre il sequestro conservativo nei confronti del coniuge o della parte dell'unione civile o di una persona accusata di omicidio dell'altro coniuge o parte dell'unione o persona a cui era legato da relazione affettiva e stabile convivenza, ove si rilevi la presenza di "figli della vittima" minorenni o maggiorenni ma non economicamente autosufficienti, ed a tutela dei figli della vittima a proposito della provvisionale nell'art 539 co. 2 bis c.p.p., così come modificato dall'art 4 1. n. 4/2018 [5].

Nel c.p.p. non sono contenute altre previsioni del concetto di "vittima": in tutti gli altri casi, si fa riferimento alla nozione di "persona offesa dal reato"o "persona danneggiata dal reato". Con il primo termine si indica il soggetto titolare dell'interesse o del bene giuridico protetto dalla norma incriminatrice, e pregiudicato o posto in pericolo dalla condotta criminosa; nel secondo caso, si indica il soggetto che ha subito un danno derivante dal reato, patrimoniale 
o non patrimoniale, in base all' art 185 c.p. e come tale è titolare della legittimazione a costituirsi parte civile all'interno del processo penale [5].

Viceversa, i riferimenti più ampi sulla nozione di vittima sono indubbiamente contenuti negli Atti internazionali.

La Risoluzione in tema di "Principi basilari di giustizia per le vittime del crimine e dell'abuso di potere", approvata il 29 novembre 1985 con la Risoluzione A/Res/ 40/34 dell'Organizzazione delle Nazioni Unite e definita un evento “... di portata storica per l'umanità, poiché ha portato la vittima al livello alto delle consacrazioni di tipo universale e cioè al livello dei diritti dell'uomo" [6], dedica vasta attenzione alle vittime del crimine.

In primo luogo, attraverso tale documento si rinviene una definizione di vittime, indicate come "le persone che, individualmente o in forma collettiva, hanno sofferto un danno, inclusa una lesione fisica o psichica, una perdita economica o una violazione sostanziale dei loro diritti fondamentali, mediante azioni od omissioni che violano le leggi penali in vigore in uno Stato membro, comprese quelle che perseguono penalmente gli abusi di potere". Nello stesso documento si sottolinea che sono vittime anche coloro i quali, in quanto familiari o in quanto adoperatisi a difesa del soggetto passivo, siano rimasti lesi o danneggiati. Viene evidenziata, in proposito, la maggiore estensione di tale concetto di vittima rispetto alla tradizionale visione giuridico formale della parte lesa, in cui il punto centrale è il patimento di un danno (fisico, mentale, economico, morale) in conseguenza del reato, con aperture alla vittimizzazione collettiva e alle vittime indirette (familiari, persone intervenute a difesa) [3].

Di conseguenza all'emanazione, da parte dell'Organizzazione delle Nazioni Unite, della Risoluzione A/Res/40/34, vengono rivolte ai singoli stati delle raccomandazioni rivolte ad incoraggiare la recezione di tali principi nei sistemi penali interni. In tale prospettiva si susseguono, da parte dell'Economic and Social Council dell'ONU, la ris. n. 1997/33 sugli "Elementi di una responsabile prevenzione della criminalità: standard e norme", approvata il 21/7/1997; la ris. n. 1998/2003 sulla "Cooperazione internazionale tesa alla riduzione del sovraffollamento delle prigioni ed alla promozione di pene alternative", approvata il 28/7/1998, e la ris. n. 1999/26 sullo "Sviluppo ed attuazione di interventi di mediazione e giustizia riparativa nell'ambito della giustizia penale", approvata il 28/7/1999 [5].

Successivamente, con la Dichiarazione di Vienna su "Criminalità $e$ Giustizia" ( $\mathrm{X}^{\circ}$ Congresso dell'ONU su "Prevenzione del crimine e trattamento dei detenuti", 10-17 aprile 2000) da parte degli Stati viene assunto l'impegno alla creazione di “... adeguati programmi di assistenza alle vittime del crimine, a livello nazionale, regionale ed internazionale, quali meccanismi per la mediazione e la giustizia riparativa".
Parimenti, si osserva come in questa materia siano numerose anche le influenze provenienti dal Consiglio d'Europa e dalla giurisprudenza della Corte europea dei diritti dell'uomo: quest'ultima, in particolare, si volge ad attribuire pari rilevanza ai diritti fondamentali di tutti i soggetti che sono coinvolti nel processo penale e, fra questi, i soggetti "deboli" quali le vittime del reato, percepite come solitamente meno tutelate in termini di garanzie e di partecipazione al processo penale ${ }^{[5]}$.

L'Unione Europea, con decisione quadro 2001 2220 GAI del 15 marzo 2001, ha provveduto alla elaborazione di una vera e propria "carta dei diritti delle vittime", che vincola gli Stati a conformare il sistema interno alle richieste dell'ordinamento sovranazionale.

Il documento in oggetto nell'articolo 1 definisce vittima la persona fisica che ha subito un pregiudizio, anche fisico o mentale, sofferenze psichiche, danni materiali causati direttamente da atti od omissioni che costituiscono una violazione del diritto penale di uno Stato membro, e ad essa fa seguito la dir. 2004/80/CE del 29 aprile 2004 che, in tema di indennizzo delle vittime di reato, definisce come vittima "una qualsiasi altra persona lesa dal reato". Analogamente, nel 2012 l'articolo 2 della direttiva 29/UE fornisce una definizione di vittima come "... una persona fisica che ha subito un danno, anche fisico, mentale o emotivo, o perdite economiche che sono stati causati direttamente da un reato", precisando che deve essere considerato vittima anche "... un familiare di una persona la cui morte è stata causata direttamente da un reato e che ha subito un danno in conseguenza della morte di tale persona" e comprendendo, inoltre, tra i familiari anche le convivenze more uxorio. Coerentemente a tali definizioni, quindi, si osserva come complessivamente nelle pronunzie internazionali venga adottato un concetto di vittima prevalentemente criminologico, i cui contorni non sono pienamente definiti sul piano giuridico e risentono dell'assenza di una coerente armonizzazione tra i sistemi dei diversi paesi che fanno parte dell'Unione Europea [5].

Dalla Corte europea dei diritti dell'uomo viene adottata, sul piano giurisprudenziale, una definizione di vittima come quel soggetto che ha subito la violazione dei diritti garantiti dalla Convenzione CEDU ed è dunque legittimato ad attivare il ricorso individuale alla Corte dei Diritti dell'Uomo previsto dall'articolo 34 CEDU. Va peraltro sottolineato come la Convenzione europea dei diritti dell'uomo non faccia alcun riferimento alla vittima quale soggetto avente un ruolo ed un diritto ad una specifica tutela in seno al processo penale.

Nel 1983 a Strasburgo in seno al Consiglio d'Europa viene firmata la Convenzione numero 116 sul risarcimento alle vittime di crimini violenti nella 
quale, inquadrando il risarcimento delle vittime come un dovere derivante da equità e solidarietà sociale, viene raccomandato ai Paesi membri di prevedere un indennizzo a carico dello Stato per le vittime di atti intenzionali violenti [4].

Da allora, numerose altre raccomandazioni del Consiglio di Europa si sono susseguite fino al 2006 a tutela della vittima e della vittima vulnerabile (Raccomandazione R(83)7; Raccomandazione R(85)11; Raccomandazione R(87)21; Raccomandazione R(97)13; Raccomandazione R(2000)19, Raccomandazione R(2006)8.

Il fine perseguito da tali documenti risiede nell'obiettivo di indirizzare le politiche penali degli Stati dell'Unione verso la tutela di questi contenuti: nella stessa prospettiva, nel 2010 entra in vigore la convenzione del Consiglio d'Europa per la protezione dei minori contro lo sfruttamento e gli abusi sessuali (Convenzione di Lanzarote), anch'essa rivolta ad uniformare la normativa dei singoli stati nella criminalizzazione di ogni forma di abuso sessuale nei confronti dei minori, anche commessi fra le mura domestiche o in famiglia con l'uso di forza, costrizione o minaccia. Analogamente, il primo agosto 2014 entra in vigore la Convenzione del Consiglio d'Europa sulla prevenzione e lotta contro la violenza nei confronti delle donne e la violenza domestica (Convenzione di Istanbul): in questo caso, l'obiettivo è quello di realizzare a tutela delle donne forme di piena protezione giuridica nei confronti di qualsiasi forma di violenza, comprese le forme di violenza domestica. In questa sede, viene altresì precisato che anche $\mathrm{i}$ bambini testimoni di violenze perpetrate all'interno della famiglia devono essere considerati vittime della violenza domestica. In attuazione delle Convenzioni di Lanzarote e di Istanbul sono state introdotte nell'ordinamento italiano leggi speciali a tutela dei diritti del minore sia come vittima che come testimone (fra queste, cfr. la 1. n. 172/2012).

Recentissima, la sentenza primo febbraio 2018 della Corte europea sui diritti dell'uomo, sez. I, condanna l'Italia per violazione degli obblighi positivi che ricadono sulle autorità giudiziarie e amministrative, consistenti nel disporre idonee misure tempestive atte a garantire la protezione dei minorenni che si trovano in una condizione di vulnerabilità sia da offese alla loro integrità psicofisica portate da soggetti terzi sia da comportamenti dannosi tenuti dai minori stessi [7].

\section{L'evoluzione della riflessione sulla vittima in criminologia ed in vittimologia}

Von Hentig - criminologo di matrice culturale giuridica - sviluppa ed amplia l'analisi della vittima elaborando ed approfondendo più nozioni, a partire da quella di "criminale-vittima" in rapporto alla quale evidenzia, in particolare, come i due ruoli possano alternarsi e/o coesistere [1]. Egli individua anche la nozione di "vittima latente", fondata sull'esistenza di una "predisposizione" al divenire vittima, sia di tipo "generale" - presente nelle "vittime nate" e nelle "vittime recidive" - sia di tipo "speciale", derivante da fattori sociali, demografici e psicopatologici. Infine, nei suoi studi viene analizzato il rapporto che si instaura tra autore di reato e vittima, all'interno del quale si possono registrare inversioni di ruolo.

A partire dallo stesso momento storico e proseguendo negli anni successivi, ulteriori sviluppi della vittimologia derivano dagli studi di Mendelsohn [8], che si rivolgono ad elaborare una riflessione di carattere generale, allargata a tutte le differenti tipologie di vittima.

In seguito il pensiero di più studiosi si rivolge ad approfondire il rapporto che esiste tra vittima ed autore di reato: Ellenberger [9], nel 1954, individua e differenzia relazioni di tipo "nevrotico puro", di tipo "psicobiologico" e di tipo "genobiologico", concepite come condizioni non necessariamente alternative ma, eventualmente, anche coesistenti.

Si evidenzia come lo stesso concetto, di "vittima che precipita il reato" [10] venga elaborato da Wolfgang nel 1958 con riferimento a reati di omicidio nei quali la vittima è la prima ad agire e ad instaurare attivamente la relazione con l'autore del reato [11], e da Amir nel 1971, in rapporto a reati di stampo sessuale nei quali la vittima è, o viene percepita dal reo, come almeno inizialmente consenziente [12].

In tema di percezione dell'atteggiamento della vittima da parte dell'autore di reato, in seguito Fattah nel 1979 mette in evidenza i processi di razionalizzazione adottati dal reo nella fase che precede il reato, rivolti ad eliminare o attenuare le resistenze a commettere il delitto e, viceversa, come in situazioni specifiche le vittime possano sviluppare percezioni positive nei confronti del loro aggressore [13].

Circa la compartecipazione che il comportamento della vittima può fornire alla realizzazione del reato, Sparks nel 1982 propone una differenziazione a partire da un comportamento di "precipitazione", e proseguendo con condotte definite di "facilitazione", di "vulnerabilità", di "opportunită" ed, infine, di vera e propria "attrattività" [14].

La diffusione nella cultura sociale degli sviluppi della vittimologia non poteva non influenzare anche il pensiero della dottrina penalistica: in particolare in Germania, la concezione di un ruolo attivo della vittima nella produzione del fatto di reato si traduce nello sviluppo di una corrente di pensiero, denominata vittimo-dommatica, che arriva a ricavarne una diretta rilevanza sulla configurabilità della fattispecie delittuosa. Pur partendo, dunque, dalle 
riflessioni della vittimologia si realizza, in tal modo, un netto allontanamento dagli obiettivi di quest'ultima - sempre rivolta a sollecitare l'attenzione a vantaggio della vittima - poiché l'impostazione vittimo-dommatica “...si pone, inequivocabilmente, dalla parte dell'agente, poiché è proprio sulla base della scoperta di una corresponsabilità della vittima nella produzione di non pochi fatti lesivi che viene a fondarsi una lettura meno ampia delle fattispecie incriminatrici corrispondenti" [4].

Ulteriori sviluppi di tale prospettiva, fondati su una concezione del diritto penale come ultima ratio - cui ricorrere solo allorché manchino altri mezzi di tutela dei beni giuridici - spostano l'attenzione, e la valutazione del giudice, sul grado di pericolo cui è esposto il bene tutelato dalla norma, sul comportamento della vittima e sulle sue possibilità di autotutela, arrivando a considerare illegittima l'applicazione del diritto penale e ritenendo che non sussista la necessità di tutela della vittima, in presenza di una sua condotta socialmente inadeguata [4].

Si sottolineano, al riguardo, le riserve dottrinali circa l'accoglibilità delle riflessioni dei vittimo-dogmatici [3], per i problemi che deriverebbero da una “... utilizzazione generalizzata dei principi di autoresponsabilità: dalla privatizzazione del diritto penale, con conseguente legittimazione di forme di autotutela, all'instaurazione di un clima sociale di sospetto e diffidenza generalizzati" [15].

Del pari, si osserva come tali tesi siano in contrasto con principi di rilevanza centrale della nostra Costituzione, quale il principio di determinatezza della fattispecie, di certezza del diritto, di eguaglianza di trattamento, senza tuttavia per ciò escludere "... del tutto il significato e la portata che la scoperta del ruolo della vittima nell'eziologia del reato può rivestire per il diritto penale sostanziale" [4].

Successivamente l'applicazione della teoria dei sistemi, e dunque lo sviluppo di un approccio sistemico alla relazione autore-vittima, ne supporta un'interpretazione in termini di causalità circolare, nella quale riveste un ruolo importante per la precipitazione del reato anche l'erronea attribuzione di intenzioni all'altro [16. 17].

L'analisi dell'influenza delle situazioni ambientali, unitamente all'attenzione per le attività e lo stile di vita del soggetto, vengono inoltre sviluppati negli studi sul "lifestyle/exposure model of victimization" [18] e sul "routine activity approach" [19], rivolti a valorizzare rispettivamente l'incidenza dello stile di vita e delle attività quotidiane della vittima sul rischio di subire un reato.

\section{Gli sviluppi del pensiero criminologico e vittimologico: la "giustizia riparativa" e la mediazione penale}

Negli ultimi decenni del 1900, inoltre, si sviluppa nella riflessione criminologica e vittimologica un orientamento di grande rilevanza ai fini dell'intervento sulla vittima del reato, la giustizia riparativa, definito come "... un processo nel quale tutte le parti hanno un interesse ad affrontare gli effetti che derivano dalla commissione di un reato si riuniscono per gestire collettivamente tali conseguenze e le loro implicazioni per il futuro" [20].

È di tutta evidenza la rilevanza che tale tipologia di intervento riveste non solo ai fini dell'ineludibile tensione all'utilizzazione dell'intervento penale quale ultima ratio, e dunque limitatamente ai soli casi nei quali non siano applicabili altri strumenti, ma anche per assicurare alla vittima un ruolo di maggiore e più significativa partecipazione alla vicenda delittuosa [21].

Anche in questo ambito, si registra un sostanziale contributo degli Atti internazionali, sviluppati in particolare dall'Unione Europea, rivolti in termini importanti allo sviluppo di un notevole potenziamento degli interventi di "mediazione" e ciò, in particolare, nel passaggio dalla Decisione quadro del 2001/220/GAI alla dir. 2012/29 UE. Si assiste infatti, a seguito di questo percorso evolutivo, al passaggio da alcune generiche previsioni circa l'obbligo per gli Stati membri di sviluppare la mediazione nei procedimenti penali per i reati ritenuti idonei, all'esplicita previsione di adeguate misure di protezione per la vittima che partecipa ai percorsi di mediazione, assicurando al contempo il suo diritto di accesso a servizi di giustizia riparativa sicuri e competenti [5].

\section{Classificazioni delle vittime nella riflessione scientifica criminologica e vittimologica}

L'approfondimento della riflessione scientifica vittimologica e criminologica ha consentito l'individuazione di differenti tipologie di vittima: viene posta una prima basilare distinzione tra vittime reali e false vittime, differenziando poi all'interno di queste ultime la vittima simulatrice e la vittima immaginaria, in funzione della presenza o meno di consapevolezza dell'inesistenza del reato [3].

Viceversa, all'interno delle vittime reali vengono individuate le c.d. vittime fungibili (prive di un rapporto con il reo, e che sono tali per eventi accidentali), o infungibili: tra le prime, è stata oggetto di specifica analisi la vittima "indiscriminata", studiata in rapporto al terrorismo [22], e successivamente approfondita anche in relazione alla delinquenza stradale [23]. 
In rapporto alle vittime infungibili vengono approfondite le c.d. "predisposizioni vittimogene", suddivise in predisposizioni biofisiologiche (quali ad esempio l'età, il sesso, le condizioni fisiche), psicologiche (strutture di personalità e tratti di imprudenza, impulsività e stati psicopatologici) e sociali (rischi connessi ad attività professionali, a condizioni di debolezza quali l'immigrazione, l'appartenenza a minoranze razziali, etc.) [3].

Parallelamente, a partire da una prima basilare distinzione tra vittime passive e vittime attive [21] vengono definite, tra le prime, la vittima accidentale (divenuta tale per caso); la vittima preferenziale (scelta per il suo ruolo o la sua posizione sociale); la vittima simbolica (scelta per colpire tutto un gruppo o una categoria) e la vittima trasversale (scelta allorché sia impossibile colpire il vero bersaglio).

Tra le vittime attive si individuano le vittime per la loro specifica professione, le vittime che aggrediscono (con riferimento agli artt. 52 e 54 c.p., "difesa legittima" e "stato di necessità"), la vittima provocatrice (connotata da un atteggiamento volontario verso l'autore di reato), la vittima favorente (in cui invece manca una vera ostilità verso l'autore di reato), la vittima consenziente (con riferimento alle fattispecie di omicidio del consenziente, di eutanasia, ed altro).

Vengono, infine, segnalate situazioni particolari sotto il profilo classificatorio, quali la sindrome di Stoccolma, in cui si instaura un rapporto positivo tra vittima ed aggressore, e le false vittime, definite vittime simulatrici in caso di menzogna consapevole, e vittime immaginarie se tale condotta deriva da immaturità o fattori psicopatologici.

\section{L'attenzione verso il minore vittima di reato nelle previsioni normative e nella riflessione scientifica criminologica}

Sotto il profilo vittimologico, data la particolare esposizione dei minori a rivestire - per l'appunto - il ruolo di vittima, il nostro impianto normativo, rispetto al minore vittima di reato, si caratterizza per una visione generale di protezione del fanciullo, riconoscendogli il diritto a crescere in un ambiente familiare che consenta l'armonioso sviluppo della sua personalità ed una serena evoluzione. I principi di tutela del minore sono presenti nella legislazione italiana a partire dalla Costituzione che, oltre ad enunciare i diritti inviolabili di cui anche il minore, in quanto persona, è titolare, delinea una concezione di famiglia come formazione sociale finalizzata alla promozione e allo sviluppo dei suoi componenti, soprattutto se minori di età. La normativa italiana riguardo i diritti dei minori in famiglia (L. n. 183/1984, L. n.149/2001) indica espressamente la necessità di basare la valutazione delle capacità genitoriali su criteri il più possibile scientifici, così da risultare maggiormente attendibili, a partire dalle evidenze disponibili [24]. Tuttavia, nonostante tale impianto normativo generale, ad esso spesso non corrisponde, concretamente, una altrettanto efficace protezione a tutela del minore. È infatti innegabile come il bambino e l'adolescente siano 'soggetti deboli', frequentemente vittime di reati di particolare gravità, spesso consumati proprio in famiglia. $\mathrm{Si}$ pensi alla violenza sessuale, alla schiavitù finalizzata allo sfruttamento, alla mercificazione, alla pornografia minorile, ai maltrattamenti, agli abusi di mezzi di correzione $[25,26]$. Aprendo il focus al contesto sociale, il minore può essere vittima del reato di violenza sessuale di gruppo, dove la spinta all'azione si rinforza in funzione della presenza dei co-offender, della condivisione e del contesto di 'squadra', spesso sotto l'effetto di alcol o droghe [27]. Sempre in ambito sociale, il minore appare particolarmente esposto a divenire vittima di reati legati all'utilizzo degli strumenti informatici, di cyberbullismo, di sostituzione di persona, di ingiurie e diffamazioni, di interferenze illecite nella vita privata, di atti persecutori. Tali fattispecie di reato, sempre più diffuse, sono facilitate dall'utilizzo degli strumenti del Web; se da un lato i minori, che ne sono fruitori numerosissimi, rappresentano una fascia nutrita come autori di reati inerenti all'utilizzo dei devices, dall'altro costituiscono una categoria particolarmente debole e bisognosa di tutela come vittime [28,29]. I reati commessi attraverso le tecnologie informatiche avanzate, infatti, sono caratterizzati da facile accessibilità, da economicità, da anonimato e possibilità di alterare l'identità, da normalizzazione e non percezione dell'agito, ricadendo questo su una vittima 'virtuale' o comunque assente, con conseguente facilitazione nella commissione di un reato che procura per la vittima, in genere minorenne a sua volta, conseguenze pericolose in quanto il vissuto, spesso gravemente mortificante e umiliante, deriva da una diffusione collettiva di contenuti attinenti la sfera personale e intima [28-31]. Queste ipotesi di reato offrono lo spunto per un raffronto critico tra le normative di altri Paesi e le scelte maggiormente tutelanti adottate dal legislatore italiano, che in tale ambito ha posto un'attenzione particolare [32]. Dal 1996 al 2006 sono state emanate in Italia diverse normative concernenti i reati a sfondo sessuale in risposta ai mutamenti culturali e sociali avvenuti con l'avvento di internet e della "globalizzazione" della comunicazione di immagini e video: la L. n. 66/1996, "Norme contro la violenza sessuale”, la L. n. 269/1998, "Norme contro lo sfruttamento della prostituzione, della pornografia, del turismo sessuale in danno di minori, quali nuove forme di riduzione in schiavitù", la L. n. 38/2006, "Disposizioni in materia di lotta contro lo sfruttamento sessuale dei bambini e la pedopornografia anche a mezzo Internet", seguita nel 2012, 
dalla succitata L. n. 172/2012, che ha ratificato la Convenzione di Lanzarote del 2007. La L. n. 71/2017 ha arricchito la tutela normativa, introducendo ulteriori specifiche disposizioni a protezione dei minori per la prevenzione ed il contrasto del fenomeno del cyberbullismo. Per alcuni reati, ad esempio la prostituzione minorile, il turismo sessuale e la detenzione di materiale pornografico minorile, è stata introdotta la perseguibilità degli autori anche per fatti commessi all'estero se compiuti da cittadini italiani, o in danno di cittadini italiani. Inoltre, in un'ottica di special prevenzione, è stato istituito un fondo per la cura delle vittime, ma anche degli autori di reato. Stanno infatti nascendo a livello capillare sul territorio nazionale diversi centri per il trattamento dell'adulto abusante, sia esso partner o genitore. A livello istituzionale centrale, sono stati introdotte misure di prevenzione e monitoraggio, quali l'istituzione presso il Ministero dell'Interno del Centro nazionale per il contrasto della pedopornografia sulla rete internet; l'istituzione dell'Osservatorio per il contrasto della pedofilia e della pornografia minorile presso il Dipartimento per le pari Opportunità della Presidenza del Consiglio dei Ministri; la creazione presso il predetto Osservatorio di una banca dati, in cui vengono inserite le azioni di contrasto alla commercializzazione di materiale pedopornografico sulla rete internet affidate alla polizia postale e giudiziaria; l'introduzione di obblighi per i fornitori dei servizi e per i fornitori di connettività delle reti di comunicazione elettronica. Nel quadro degli interventi specifici a tutela dei minorenni vittime di reati a sfondo sessuale, è opportuno ricordare la Carta di Noto, redatta nel giugno 1996; essa contiene importanti indicazioni per l'ascolto del minore vittima durante il procedimento giudiziario, facendo riferimento alle modalità tecnico-operative per l'acquisizione della testimonianza in caso di abuso sessuale e alla necessaria presenza di un operatore specializzato e specificatamente formato in grado di fornire assistenza psicologica al minore e di tutelarne la delicata sfera psicologica ed emotiva. Un rafforzamento nella tutela dei diritti dei minori in tale ambito discende, ancora una volta, dalla legge $1^{\circ}$ ottobre 2012, n. 172, nella quale il rafforzamento della tutela e della protezione giuridica dei minori vittime di reato viene effettuato attraverso l'introduzione di più misure. L'art. 4, comma 1, lettera v) - modificando l'art. 609 decies del codice penale sulla tutela del minore vittima di delitti contro la libertà sessuale - estende quanto previsto dall'art. 11 , comma $3^{\circ}$, della legge 66/1996 - sulla garanzia per il minore di assistenza da parte dei Servizi, accanto a quella dei genitori o altre persone idonee - anche ai reati previsti dagli artt. 600, "Riduzione o mantenimento in schiavitù o servitù ", 600 bis "Prostituzione minorile", 600 ter "Pornografia minorile", 600 quinques "Iniziative turistiche volte allo sfruttamento della prostituzione minorile", 601 "Tratta e commercio di persone", 602 "Acquisto o alienazione e commercio di schiavi" del codice penale oltre che a quelli già previsti dall'art. 609 decies "Adescamento di minorenni". L'art. 5, lettere c), d) ed f), modificano vari articoli del c.p.p., prevedendo, nei procedimenti per delitti di sfruttamento sessuale di minori, di tratta di persone, di violenza sessuale e di adescamento di minori, che l'assunzione delle informazioni da minorenni avvenga con l'ausilio di un esperto in psicologia $o$ in psichiatria infantile, nel corso delle indagini preliminari, rispettivamente dalla polizia giudiziaria, dal pubblico ministero, dal difensore. Nonostante l'introduzione di tali previsioni normative, lo sfruttamento di minori in ambito sessuale mantiene una marcata caratterizzazione quale reato a diffusione capillare, di difficile individuazione e connotato da accentuate difficoltà nelle strategie di contrasto, che si confrontano con fattori di influenzamento radicati nella cultura sociale.

Si rilevano, inoltre, situazioni passibili di vittimizzazione del minore certamente sui generis: fra queste, ad esempio ed in primis, quelle in cui egli appartiene ad un contesto socio-familiare di stampo sotto-culturale, soprattutto all'interno di situazioni inerenti alla criminalità organizzata. Tali contesti, sovente caratterizzati da una rigida struttura e sottocultura delinquenziale, veicolano regole e modelli non conformi, che possono facilitare l'interiorizzazione da parte del minore di tali principi: l'agito deviante ed anti-normativo può derivare, in tali casi, dall'introiezione da parte del minore di modelli di tipo antisociale ai quali è difficile sottrarsi. È infatti evidente l'estrema difficoltà, per un minore ed un adolescente, di mettere in discussione l'assetto culturale di derivazione, di contrapporsi alle figure genitoriali e al proprio gruppo sociale. In tale ottica, sono sorte a tutela dei minori appartenenti a famiglie della criminalità organizzata, percorsi istituzionali di supporto e di sostegno [33]. A titolo esemplificativo, ricordiamo l'Associazione "Libera", che si rivolge ad individuare percorsi educativi per minori condannati per il reato di associazione di tipo mafioso. In tale prospettiva, vengono strutturati progetti di tipo extra-murario nei quali si tutelano, insieme ai minori, i membri del nucleo familiare estranei al contesto criminale, in genere madri e fratelli, a loro volta letti come 'vittime' di un sistema altamente pericoloso e uncinante, dal quale sarebbe difficilissimo affrancarsi. Tali iniziative tuttavia rappresentano ancora una risposta di carattere sperimentale, inidonea a fronteggiare un fenomeno che richiede ben altri investimenti e risorse, stante la sua elevata diffusione, ormai presente a livello capillare nel nostro territorio.

Analogamente, l'appartenenza del minore ad un nucleo familiare caratterizzato dalla presenza di relazioni e dinamiche familiari disfunzionali può costituire e viene riconosciuta come una situazione facilitante l'insorgenza 
di condizioni di disagio e di devianza, a loro volta possibili precursori della criminalità ${ }^{[21]}$. Si evidenzia, in tal senso, il rapporto che può svilupparsi tra inadeguatezza comportamentale e, talvolta, condotta anti-normativa del giovane e la sua appartenenza ad un contesto familiare disfunzionale e/o disgregato, non necessariamente connotato da deprivazione, a meno di non includere nel concetto di "deprivazione" anche l'aspetto affettivo, dell'accudimento, della cura $[21,34,34-40]$. A tal proposito, si segnalano come recente oggetto di riflessione anche fenomeni, come quello registrato nell'ultimo periodo presso il Tribunale per i Minorenni dell'Emilia-Romagna, di un significativo incremento di maltrattamenti in famiglia ad opera di minorenni - caratterizzati da un'età particolarmente giovane - che agiscono verso i genitori aggressioni verbali e fisiche di forte intensità. L'impianto normativo vigente, rivolto ad un approccio interpretativo dell'agito criminoso del minore e ad una sua tutela sotto profili sostanziali, rimanda ad una lettura olistica del giovane autore di reato. Proprio alla luce dei principi delle fonti internazionali in materia di minori - che esercitano un'azione propulsiva orientata allo sviluppo di una legislazione uniforme, anche sotto il profilo processuale - il D.P.R. 448/88 sul processo penale minorile, corpus iuris ad hoc, risulta a tutt'oggi significativamente moderno con il suo focus sulla tutela e sulla centralità del minore, finalizzato ad implementarne le risorse e supportarne le fragilità. Si osserva, in tal senso, una sintonia con il principio generale della specificità del minore, riconosciuta per la prima volta nella Dichiarazione di Ginevra sui diritti del bambino del 1924, in base alla quale si ritiene che la peculiarità dell'individuo minore richieda particolari tutele. E ciò soprattutto in materia penale, anche in prospettiva preventiva nei confronti dei fenomeni della devianza, della criminalità giovanile e della riduzione della recidiva. Sono significative, in tal senso, le Regole di Pechino, adottate dall'ONU nel 1985, confluite nella Convenzione dell'ONU sui diritti del fanciullo del 1989 - ratificata dall'Italia con la L. 176/1991 - che identificano nel minore un soggetto di diritti, titolare di posizioni giuridiche autonome e distinte da quelle del nucleo familiare di appartenenza, portatore del diritto di essere ascoltato in ogni processo decisionale che lo riguardi, e che dedicano ampio spazio al tema della giustizia minorile.

\section{Riflessioni conclusive}

L'evoluzione raggiunta dalla riflessione scientifica nella criminologia e nella vittimologia può e deve rinforzare i moderni obiettivi della politica criminale nel rivolgersi a garantire nel processo penale l'armoniosa tutela dei diritti dell'imputato e di quelli della vittima. Ciò ch'è essenziale, in questa prospettiva, è la consapevolezza che una maggiore partecipazione di quest'ultima non deve essere interpretata e non deve tradursi in un arretramento della tutela del primo, protetto da principi costituzionali e sovranazionali, quali la presunzione d'innocenza, il diritto di difesa, il diritto ad un fair trial. Al contrario, è essenziale la consapevolezza che la coesistenza delle due posizioni non va vissuta in forma di antitesi tra le stesse ma, viceversa, nella prospettiva della pari dignità dei due ruoli [5].

Analogamente, è importante che la riflessione scientifica supporti la consapevolezza dell'opportunità di non cadere in reazioni emotive, così frequenti nell'opinione pubblica e sovente rinforzate dai mass-media, che supportano la percezione che le attenzioni ai diritti degli autori di reato si traducano in una riduzione dell'attenzione e del rispetto verso la vittima, non potendosi dimenticare in tale ambito l'imprescindibilità di una attenta tutela dei diritti di entrambe le parti escludendo, parallelamente, ogni atteggiamento di prepotenza e di abuso [21].

Se queste indicazioni in termini di politica criminale e de jure condendo rivestono rilievo per la vittima adulta, ciò vale in termini ben più incisivi nei confronti del minore, per definizione l'elemento più fragile e più prezioso all'interno del gruppo sociale, i cui percorsi di crescita, di educazione e di sviluppo costituiscono un obiettivo irrinunciabile.

\section{Bibliografia}

[1] Hentig V. H. The Criminal and his Victim, Studies in Sociobiology of the Crime. New Haven: Schocken Books; 1979.

[2] Gulotta G. Elementi di psicologia giuridica e di diritto psicologico. Milano: Giuffrè; 2000.

[3] Martucci P. Vittima del reato. Enciclopedia giuridica aggiornamento. 2003; XLIV: 593-596.

[4] Del Tufo V. Vittima del reato. Enciclopedia del diritto. 1993; XLVI: 996-1003.

[5] Montagna M. Vittima del reato (Profili processuali). Digesto. Torino: UTET; 2018; 962-966.

[6] Pisani M. Per le vittime del reato. Rivista italiana di diritto e procedura penale. $1989 ; 467$.

[7] Sibilio D. Secondo la Corte europea lo Stato ha il dovere di proteggere i minori vulnerabili anche da se stessi. Rivista italiana di diritto e procedura penale. 2018; 980-984. 
[8] Mendelsohn B. Victimology ad contemporary society's trends. General victimology. Victimology an international journal. 1977; 16-18.

[9] Ellenberger H. F. Relations psychologiques entre le criminal et la victim. Revue international de criminology et de police technique. 1954; 8: 103-106.

[10] Bandini T. Vittimologia. Enciclopedia del diritto. XLVI; 1993; 1009-1015.

[11] Wolfgang M. E. Patterns in criminal homicide. Philadelphia: University of Pennsylvania press; 1958.

[12] Amir M. Patterns in forcible rape. Chicago: University of Chicago press; 1971.

[13] Fattah E.A. Some recent theoretical developments in victimology. Victimology. 1979; 198-207.

[14] Sparks R. F. Research on victims of crime: accomplishments, issues and new directions. National institute of mental healt (U.S.). Rockville: University of California libraries; 1982.

[15] Cagli S. Condotta della vittima e analisi del reato. Rivista italiana di diritto e procedura penale. $2000 ; 1148-1158$.

[16] Gulotta G, Vagaggini M. Dalla parte della vittima. Milano: Giuffrè; 1980.

[17] Gulotta G, De Cataldo Neuburger L. A systemic and attributional approach to victimology. International journal of victimology; 1983, 5: 16-21.

[18] Hindelang M.J, Gottfredson M.R, Garofalo J. Victims of personal crime: an empirical foundation for a theory of personal victimization. Cambridge: Ballinger; 1978.

[19] Cohen L.E, Felson M. Social change and crime rate trend: a routine activity approach. American sociological review. 1979; 588-593.

[20] Marshall T.F. Restorative justice. An overview. Buchkinghamshire: Home office; 1999. Ponti G, Merzagora I. Compendio di criminologia, Milano: Raffaello Cortina

[21] Editore; 2008, 548-558.

Nuvolone P. La vittima nella genesi del delitto. Indice penale. 1973; 3: 640-642.

[22] Mantovani F. Il problema della criminalità. Padova: Cedam; 1984, 386-393.

[23] Camerini G. B, Volterra V. Criteri di valutazione delle capacità genitoriali e

[24] strumenti operativi. Rassegna italiana di criminologia 2007; 3: 135-152. Bertolino M. Il minore vittima di reato. Torino: Giappichelli; 2010.

[25] Gualco B, Rensi R, Fossa G. Violenza assistita e comportamenti devianti dei

[26] giovani in Italia: i risultati dell'International self-report delinquency study-3. Rassegna italiana di criminologia. 2017, 2: 104-114.

Zara G, Veggi S. I minorenni sessualmente abusanti tra solo-offending e co-

[27] offending. Uno studio operativo. Rassegna italiana di criminologia. 2018, 4: 262-274.

Caffò A, Catanesi R, Greco R, Grattagliano I, Toma E, Taurino A, Bosco A.

[28] Cyberbullismo: nuova forma di bullismo o specifica manifestazione di violenza sul Web? Rassegna italiana di criminologia, 2017, 1,76-82.

Baldry A. C, Sorrentino A. Il cyberbullismo, una nuova forma di disagio

[29] giovanile. Rassegna italiana di criminologia. 2013, 4: 264-276.

[30] De Fazio L, Sgarbi C. Nuove prospettive di ricerca in materia di atti persecutori: il fenomeno del cyberstalking. Rassegna italiana di criminologia. 2012, 3: 146-159.
[31] Caffò A, Catanesi R, Greco R, Grattagliano I, Toma E, Taurino A. Il ruolo di internet e degli strumenti informatici di comunicazione sulla qualità delle relazioni tra preadolescenti. Una ricerca pilota. Rassegna italiana di criminologia. 2017, 1: 67-75.

[32] Conti A. Le garanzie processuali del minore: un confronto tra l'ordinamento europeo e la disciplina italiana. Minorigiustizia. 2019, 2: 96-107.

[33] Schermi M. L'educazione criminale: crescere in contesti mafiosi. Rassegna italiana di criminologia. 2013. 4: 256-263.

[34] Caruso M G, Cerbara L, Tintori A. Stereotipi, bullismo e devianza a scuola Identikit degli studenti italiani. Minorigiustizia. 2019, 2: 133-141.

[35] Gatti U. Rocca G. Verde A. Devianza giovanile e giustizia minorile, fra tradizione e cambiamento. Rassegna italiana di criminologia. 2013, 4: 234-235.

[36] Rocca G. Gatti U. I programmi di trattamento psicosociale dei disturbi della condotta in età evolutiva al vaglio della ricerca valutativa. Rassegna italiana di criminologia. 2013, 4: 296-307.

[37] Susanna Pietralunga, Claudia Salvioli, Ivan Galliani. Reati violenti commessi da minorenni. La vulnerabilità biologica, psichica e sociale del minore. Rassegna italiana di criminologia. 2010, 2: 237-253.

[38] Bandini T, Gatti U, Gualco B, Malfatti D, Marugo M I, Verde A. Criminologia, il contributo della ricerca alla conoscenza dl crimine e della reazione sociale. Milano: Giuffré Editore; 2004. p. 141-187, 539-557.

[39] Gualco B, Fossa G, Malagoli, Orlandi E, Salvadori L, Rensi R, Presenza dei genitori e vittimizzazione dei giovani in Italia. Risultati dell'International Selfreport Delinquency Studi-3, Rassegna italiana di criminologia. 2019, 1: 30-38.

[40] Sabatello U, Giacolini T, Thomas F. Condotte aggressive e antisociali nell'infanzia e nell'adolescenza: alcune riflessioni cliniche e psicopatologiche. Rassegna italiana di criminologia. 2018, 3: 231-246.

[41] Spadaro G. La giurisdizione minorile ed i soggetti vulnerabili. Minorigiustizia. 2019, 1: 51-57.

\section{Per corrispondenza}

Email: susanna.pietralunga@unimore.it 